$$
\begin{gathered}
\text { CONF- } 9507 / 75--る \\
\text { UCRL-JC-124249 } \\
\text { PREPRINT }
\end{gathered}
$$

\title{
Quantitative XRFA of Carbon in a Special Matrix by the Fundamental Parameter Method
}

\author{
F. A. Weber, L. B. Da Silva, T. W. Barbee, Jr., \\ D. Ciarlo, M. Mantler
}

\section{RECENED \\ JUN 27 1996 \\ OSTI}

This paper was prepared for submittal to the

44th Annual Denver X-ray Conference

Colorado Springs, CO

July 31-August 4, 1995

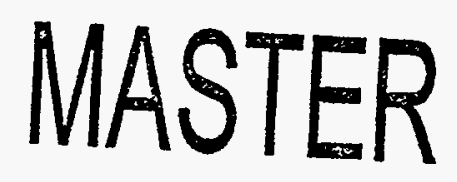

May 1996

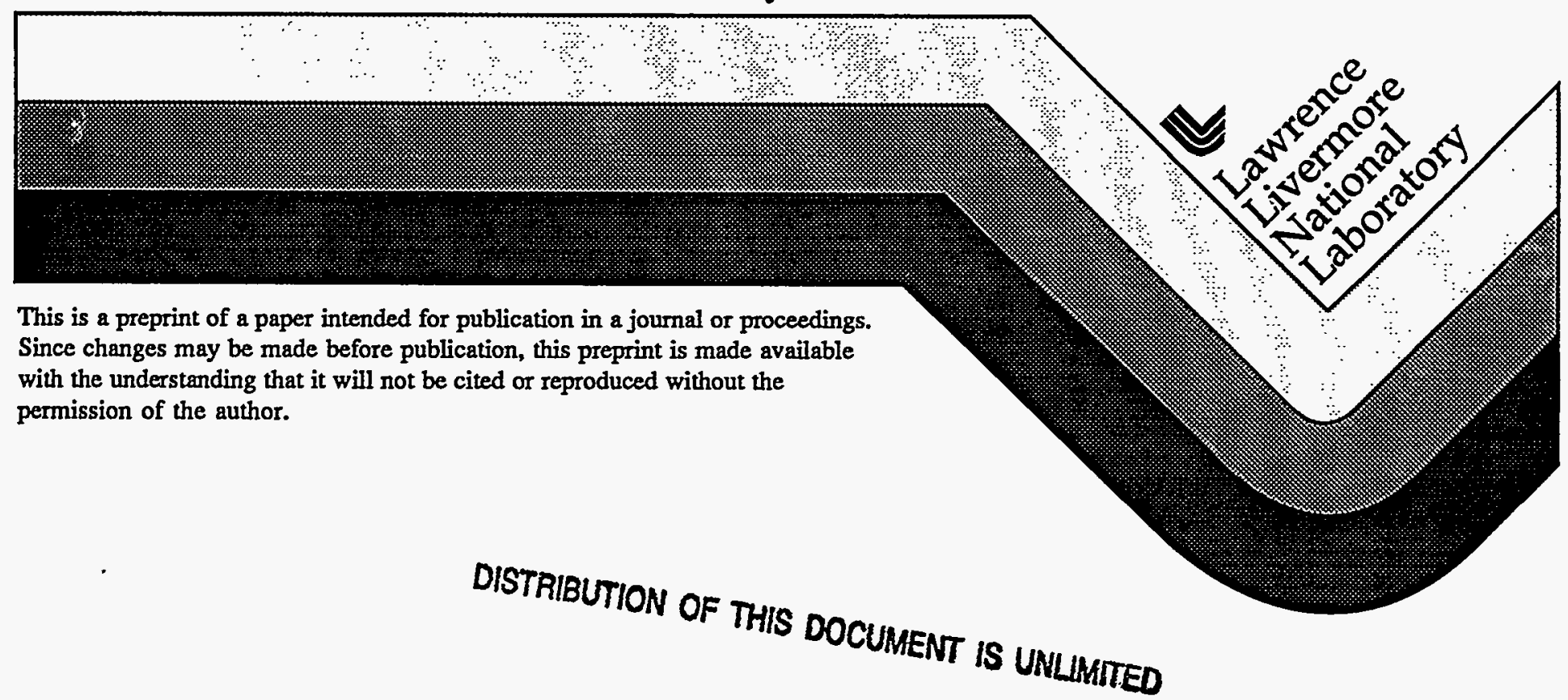




\section{Disclaimer}

This document was prepared as an account of work sponsored by an agency of the United States Government. Neither the United States Government nor the University of California nor any of their employees, makes any warranty, express or implied, or assumes any legal liability or responsibility for the accuracy, completeness, or usefulness of any information, apparatus, product, or process disclosed, or represents that its use would not infringe privately owned rights. Reference herein to any specific commercial products, process, or service by trade name, trademark, manufacturer, or otherwise, does not necessarily constitute or imply its endorsement, recommendation, or favoring by the United States Government or the University of California. The views and opinions of authors expressed herein do not necessarily state or reflect those of the United States Government or the University of California, and shall not be used for advertising or product endorsement purposes. 


\title{
QUANTITATIVE XRFA OF CARBON IN A SPECIAL MATRIX
}

\section{BY THE FUNDAMENTAL PARAMETER METHOD}

\author{
Franz A. Weber, Luiz. B. Da Silva, Troy W. Barbee, Jr., and Dino Ciarlo \\ University of California, Lawrence Livermore National Laboratory, \\ P.O. Box 808, Livermore, CA 94551, USA
}

\section{Michael Mantler}

Institute of Applied and Technical Physics

Technical University Vienna, A-1040 Vienna, Austria

\section{SUMMARY}

We report on results obtained from experiments using specially prepared carbon substrates and treatment of the data by means of recently introduced theory. ${ }^{1}$ Medium $\mathrm{Z}$ grids with known parameters have been coated on top of pyrolytic carbon substrates to achieve well defined absorption geometries. The various copper grids exhibit satisfactory performance in terms of mechanical stability, homogeneity and uniformity of the coating. A detailed study of the measurement results shows that there is a more rapid increase of the associated $\mathrm{C}-\mathrm{K} \alpha$ countrate from the coated samples compared to the pure elements and is attributed to the contribution of secondary enhancement effects, including those resulting from photoelectrons generated after the primary ionization.

A variety of multilayer analyzers has also been evaluated during these experiments. Only a certain combination of multilayer component materials have been found to be appropriate for use as dispersing elements due to the reflectivity and spatial resolution requirements of our long wavelength spectrometer. Another experimental factor is the low intensity of available tube photons which is due to the selection of the target material and absorption effects in the target as well as the tube window.

\section{INTRODUCTION}

Quantitative analyses of samples containing low $\mathrm{Z}$ elements such as carbon and boron based on Xray fluorescence analysis (XRFA) require modifications of the standard fundamental parameter model $^{2}$. One possible source of inaccuracy and in turn, a fundamental parameter disadvantage when 
compared to empirical and semiempirical coefficient methods ${ }^{3}$ in the low energy region, is the existence of secondary and higher order enhancement effects as they have considerable influence on the quantification procedures which relate measured and properly processed countrates to concentrations of elements of interest in the specimen.

However, the implementation of secondary enhancement effects and in particular effects due to photoelectrons are not available in commercially available software packages. Therefore, we have undertaken a series of experiments intended to determine the magnitude of such effects in both pure element and compound specimens containing low atomic number materials.

\section{THEORETICAL APPROACH}

Based on the work of Shiraiwa and Fujino ${ }^{4}$ the number of observed fluorescence photons originating from a certain transition in an element of interest within a specimen can be described as the sum of counts from primary and secondary excitation

$$
n_{i}=n_{i, p r i m}+n_{i, s e c}
$$

where $\mathrm{i}$ denotes the analyte line emitted by the element of interest. The quantities $n_{i, p r i m}$ and $n_{i, s e c}$, respectively, can be expressed as a function of the element's fundamental parameters and the excitation integral with

$$
n_{i, \text { prim }}=\text { const } \cdot \frac{\Omega}{4 \pi} \cdot \kappa_{i} \cdot \omega_{i} \cdot \frac{S_{i}-1}{S_{i}} \cdot p_{i} \cdot c_{i} \cdot \int_{\lambda_{0}-c d s e}^{\lambda_{i_{-}}}-\frac{\tau_{i}(\lambda) \cdot I(\lambda) \cdot d \lambda}{\frac{\mu(\lambda)}{\sin \psi_{1}}+\frac{\mu(i)}{\sin \psi_{2}}}
$$

and

$$
\begin{aligned}
n_{i, \mathrm{sec}}= & \operatorname{const} \cdot \frac{\Omega}{8 \pi} \cdot \kappa_{i} \cdot \omega_{i} \cdot \frac{S_{i}-1}{S_{i}} \cdot p_{i} \cdot c_{i} \cdot \omega_{j} \cdot \frac{S_{j}-1}{S_{j}} \cdot p_{j} \cdot c_{j} \cdot \int_{\lambda_{0}}^{\lambda_{\text {JK_adse }}} \frac{\tau_{i}(j) \cdot \tau_{j}(\lambda) \cdot I(\lambda)}{\frac{\mu(\lambda)}{\sin \psi_{1}}+\frac{\mu(i)}{\sin \psi_{2}}} \\
& \left\{\frac{\sin \psi_{1}}{\mu(\lambda)} \cdot \ln \left[1+\frac{\mu(\lambda)}{\mu(j) \cdot \sin \psi_{1}}\right]+\frac{\sin \psi_{2}}{\mu(i)} \cdot \ln \left[1+\frac{\mu(i)}{\mu(j) \cdot \sin \psi_{2}}\right]\right\} \cdot d \lambda
\end{aligned}
$$

The constant in equations 2 and 3 includes the geometrical factors, $\Omega$ denotes the solid angle covered by the detection system, $\kappa$ is the detector efficiency, $\omega$ the fluorescent yield, $S$ the absorption edge jump, $\mathrm{p}$ the transition probability for the line of interest, $\mathrm{c}$ the weight fraction of the element of interest, $\tau$ is the mass-photoabsorption coefficient, $\mu$ the total mass-absorption coefficient, $\mathrm{I}(\lambda) \cdot \mathrm{d} \lambda$ the number of source photons per $\mathrm{cm}^{2}$ per second at the specimen surface, and $\psi_{1}$ and $\psi_{2}$ are the angles of the incident and fluorescence photons, respectively; $i$ as an index relates a parameter to the emission process and properties of primary fluorescence radiation, and, accordingly, $j$ to secondary fluorescence radiation.

Equations 2 and 3 ordinarily apply for elements with absorption edge energies in the range above approximately $800 \mathrm{eV}$ to $1 \mathrm{keV}$ and if, in addition, homogeneous and bulk specimens (no thin films) with flat surfaces are assumed which means that the spatial dimensions are large compared to the 
information depth (i. e., depth from which $99 \%$ of the associated pure element countrate can still be detected).

Unfortunately, a number of problems arises in the long wavelength region (certainly above $\sim 44 \AA$ ) which are mainly due to the physical properties of the ultralight elements. First and foremost, low $\mathrm{Z}$ elements have very low and inaccurate and often poorly known fluorescent yields in the range of $10^{-3}$ whereas for comparison the $\mathrm{K}$-shell fluorescent yields for heavy elements are close to unity. Secondly, as the excitation integrals in equations 2 and 3 show (consider terms of the form $\tau(\lambda) \cdot I(\lambda) \cdot d \lambda)$, the intensity of the relevant carbon $K \alpha$ fluorescence radiation in the current experiments is governed by the product of the excitation spectrum (primary tube spectrum) and the absorption coefficient for carbon. In order to obtain high fluorescence intensities both factors $\tau(\lambda)$ and $I(\lambda)$ must have sufficiently large values for an extended range on the wavelength scale. Published tube spectra 5 , however, do not show any reasonable intensities from about $5 \AA$ up to the carbon absorption edge at $43.8 \AA$. Consequently, only a comparatively small number of carbon fluorescence photons is created by absorption of high energy tube photons. The rather large difference in energy between the tube photon and the absorption edge energy of carbon is then transferred to the photelectron generated after the primary ionization event and ejected from the $\left(K_{-}\right)$shell thereafter. This effect is likely to give rise to additional excitation of carbon $K \alpha$ photons within the sample and can quantitatively be taken into account in the standard fundamental parameter model by means of the following equations:

$$
n_{i, p r i m+e}=\operatorname{const} \cdot \frac{\Omega}{4 \pi} \cdot \kappa_{i} \cdot \omega_{i} \cdot \frac{S_{i}-1}{S_{i}} \cdot p_{i} \cdot c_{i} \cdot \int_{\lambda_{0}}^{\lambda_{i_{-} \text {edse }}} \frac{M_{e}\left(\lambda^{*}\right) \cdot \tau_{i}(\lambda) \cdot I(\lambda) \cdot d \lambda}{\frac{\mu(\lambda)}{\sin \psi_{1}}+\frac{\mu(i)}{\sin \psi_{2}}}
$$

where

$$
M_{e}\left(\lambda^{*}\right)=1+n_{e \rightarrow p h} \quad \text { (5a) } \quad \frac{1}{\lambda^{*}}=\frac{1}{\lambda_{\text {edge }}}-\frac{1}{\lambda}
$$

and

$$
n_{e->p h}=-\omega \cdot \frac{R \cdot N}{A} \cdot \rho \cdot \int_{E=E_{c}}^{E=E_{0}} Q \cdot d s=-\omega \cdot \frac{R \cdot N}{A} \cdot \int_{E=E_{c}}^{E=E_{0}} \frac{Q \cdot d E}{d E / d \rho s}
$$

Equation 6 describes the excitation of (characteristic) $x$-rays by electrons and has basically been derived by Green and Cosslett ${ }^{6}$. Therein $n_{e->p h}$ is the number of produced $x$-ray photons, $N$ Avogardos' number, $A$ the atomic weight, $\omega$ the fluorescent yield, $\rho$ the density, and $Q$ the ionization cross-section for the observed shell. The electron has an initial energy $E_{o}$ which it looses along its path $s$ (among other possibilities of interaction) by ionizing atoms in the shell of interest, as long as its energy exceeds their $\mathrm{K}$-absorption edge energy denoted as $\mathrm{E}_{\mathrm{C}}$. The backscattering factor, $\mathrm{R}$, is assumed to be 1 , as the photoelectrons are generated already within the specimen. However, a certain loss of photoelectrons has to be expected in case of highly absorbing materials where photoelectrons are generated within the top surface layer. If appropriate expressions for the associated ionization cross-section $Q$ and the stopping power relation $\mathrm{dE} / \mathrm{d} \rho \mathrm{s}$ are utilized the quantification of the secondary enhancement effect due to photoelectrons can conveniently be summarized by the factor $M\left(\lambda^{*}\right)$ given in equation 5a. Because of the inherently multiplicative nature of the effect the term $M\left(\lambda^{*}\right)$ enters in the numerator in the excitation integral of equation 4 which now gives the number of observed fluorescence photons excited by primary (tube) photons as well as photoelectrons. Various 
cross-sections and stopping power relations have been examined in terms of their applicability and the results are published elsewhere.

Other possibilities of unusual secondary excitation effects include Auger electrons as well as $\mathrm{L}$ alpha photons after $\mathrm{K}$-shell ionization. Along with each primary $\mathrm{K}$-shell ionization in a heavy matrix element (and K-photon emission with probability $\omega_{K}$ ) a cascade of further transitions is initiated with a high probability for an $\mathrm{L}_{2}$ or $\mathrm{L}_{3}$-shell vacancy. Each $\mathrm{K}$-photon is therefore followed by an $\mathrm{L}$-photon with account to a proper $\omega_{\mathrm{L}}$, which is much smaller than $\omega_{\mathrm{K}}$. If the L-line energy, however, lies in the vicinity of a light element's $\mathrm{K}$-absorption edge energy, secondary enhancement will be noticable. These contributions are (note that the factors $\omega_{j} \cdot p_{j}$ and $\left(S_{j}-1\right) / S_{j}$ have to be split up and that the mass-photoabsorption coefficient $\tau_{\mathrm{i}}$ changes accordingly):

$$
\begin{aligned}
& n_{i, s e c}=\text { const } \cdot \frac{\Omega}{8 \pi} \kappa_{i} \cdot \omega_{i} \cdot \frac{S_{i}-1}{S_{i}} \cdot p_{i} \cdot c_{i} \cdot \omega_{j K} \cdot \omega_{j L} \cdot \frac{S_{j K}-1}{S_{j K}} \cdot \frac{S_{j L}-1}{S_{j L}} \cdot p_{j K} \cdot p_{j L} \cdot c_{j} \cdot \\
& \int_{\lambda_{0}}^{\lambda_{j K_{-} d s c}} \frac{\tau_{i}(j L) \cdot \tau_{j}(\lambda) \cdot I(\lambda)}{\frac{\mu(\lambda)}{\sin \psi_{1}}+\frac{\mu(i)}{\sin \psi_{2}}} \cdot\left\{\frac{\sin \psi_{1}}{\mu(\lambda)} \cdot \ln \left[1+\frac{\mu(\lambda)}{\mu(j L) \cdot \sin \psi_{1}}\right]+\frac{\sin \psi_{2}}{\mu(i)} \cdot \ln \left[1+\frac{\mu(i)}{\mu(j L) \cdot \sin \Psi_{2}}\right]\right\} \cdot d \lambda
\end{aligned}
$$

In summary, equation 7 takes into account the secondary excitation of $i$ fluorescence radiation by $\mathrm{jL}$ fluorescence radiation following the initial $\mathrm{K} \alpha$ emission. We asssume that all the above mentioned effects are particularly important in case of analyses of light elements in heavy matrices since there is a large number of characteristic lines contributing to secondary enhancement. Consequently, any possible subshell effects have to be treated separately. Enhancement effects that actually take place in the specimens selected for the current experiments (and described in greater detail in the next paragraph) are compiled in table I.

\begin{tabular}{|l||c|c|c|c|c|c|c|c|}
\hline Shell ionized & $\mathrm{Cu}-\mathrm{K}$ & $\mathrm{Cu}-\mathrm{L}$ & $\mathrm{Cu}-\mathrm{K}$ & $\mathrm{Cu}-\mathrm{K}$ & $\mathrm{Cu}-\mathrm{K}$ & $\mathrm{Cu}-\mathrm{L}$ & $\mathrm{Cu}-\mathrm{K}$ & $\mathrm{C}-\mathrm{K}$ \\
\hline Excitation of & $\mathrm{Cu}-$ & $\mathrm{Cu}-$ & $\mathrm{Cu}-$ & $\mathrm{Cu}-\mathrm{K}$ & $\mathrm{Cu}-$ & $\mathrm{Cu}-\mathrm{L}$ & $\mathrm{Cu}-\mathrm{L}$ & $\mathrm{C}-\mathrm{K}$ \\
$\mathrm{C}-\mathrm{K} \alpha$ by & $\mathrm{K} \alpha$ & $\mathrm{L} \alpha$ & $\mathrm{L} \alpha$ & ph.e. & Auger e. & ph.e. & ph.e. & ph.e. \\
\hline
\end{tabular}

Table I: Enhancement effects in a Cu-C system. Primary ionization is caused by tube photons.

Compton scattering has been investigated ${ }^{8}$ and is negligible for low photon energies.

Finally frequent line overlaps, even in the first order (and depending on the design of the spectrometer), of lines of heavier matrix elements have to be considered. The average peak width in the long wavelength region is several degrees which requires the examination of relatively wide windows ${ }^{9}$. Furthermore, there are very different information depths due to different absorption properties of the sample itself. 
(a)

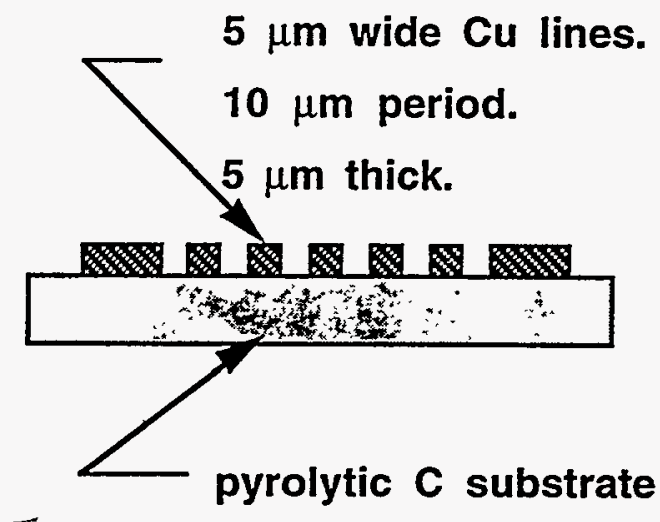

(b)

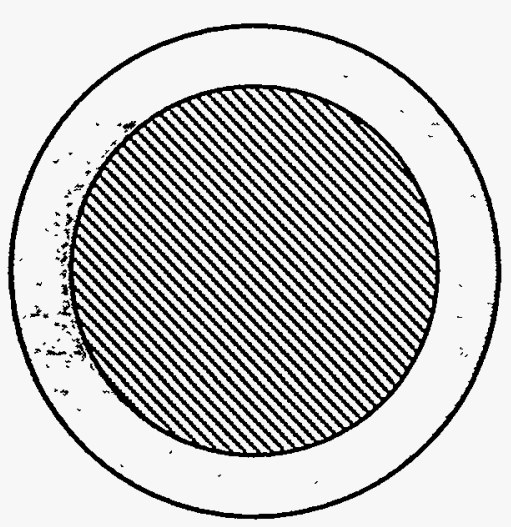

Figure 1: (a) Cross-section of the specimen. (b) Plane view of the specimen. The medium $Z$ lines occupy a central area, $33.0 \mathrm{~mm}$ in diameter.

In order to obtain a well-defined absorption geometry with accurately known active volumes for secondary excitation caused by photoelectrons and still maintain a capability of detecting a statistically sufficient number of soft $X$-rays, we envisioned a specimen design as shown in figure 1 . The target consists of an array of lines of a medium $\mathrm{Z}$ element, anywhere from $0.5 \mu \mathrm{m}$ to $5 \mu \mathrm{m}$ wide and $5 \mu \mathrm{m}$ thick, with a period of $10 \mu \mathrm{m}$. This array is on top of a pyrolytic carbon substrate, which is $1.4 \mathrm{~mm}$ thick, with a diameter of $38.1 \mathrm{~mm}$. When in the sample holder, only the central area with a diameter of $33.0 \mathrm{~mm}$ is exposed so the medium $\mathrm{Z}$ array is confined to this smaller area as shown.
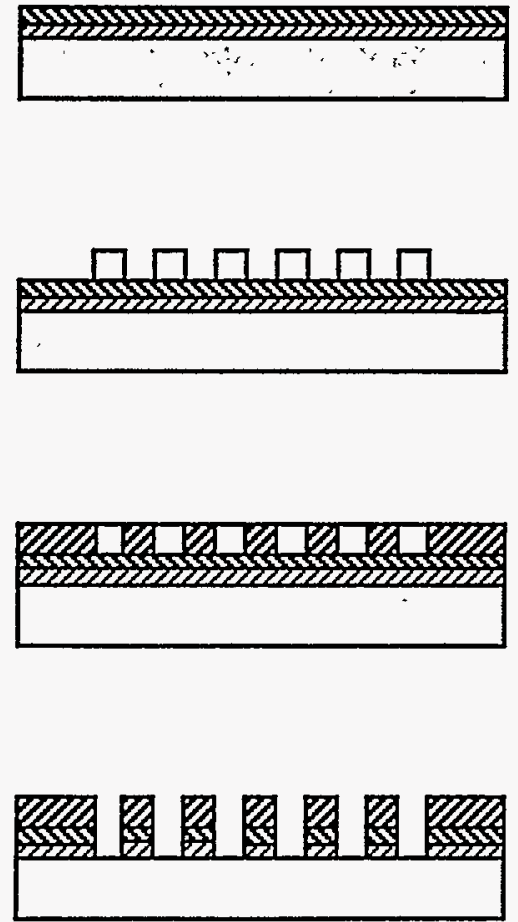

\section{a. Evaporate $100 \AA \mathrm{Cr}$ and $2000 \AA \mathrm{Cu}$ for a seed layer.}

b. Define a photoresist pattern for use as a plating mold.

c. Electroplate $\mathrm{Cu}$. seed layer.

Figure 2: Fabrication steps for the copper array on pyrolytic carbon substrate. 

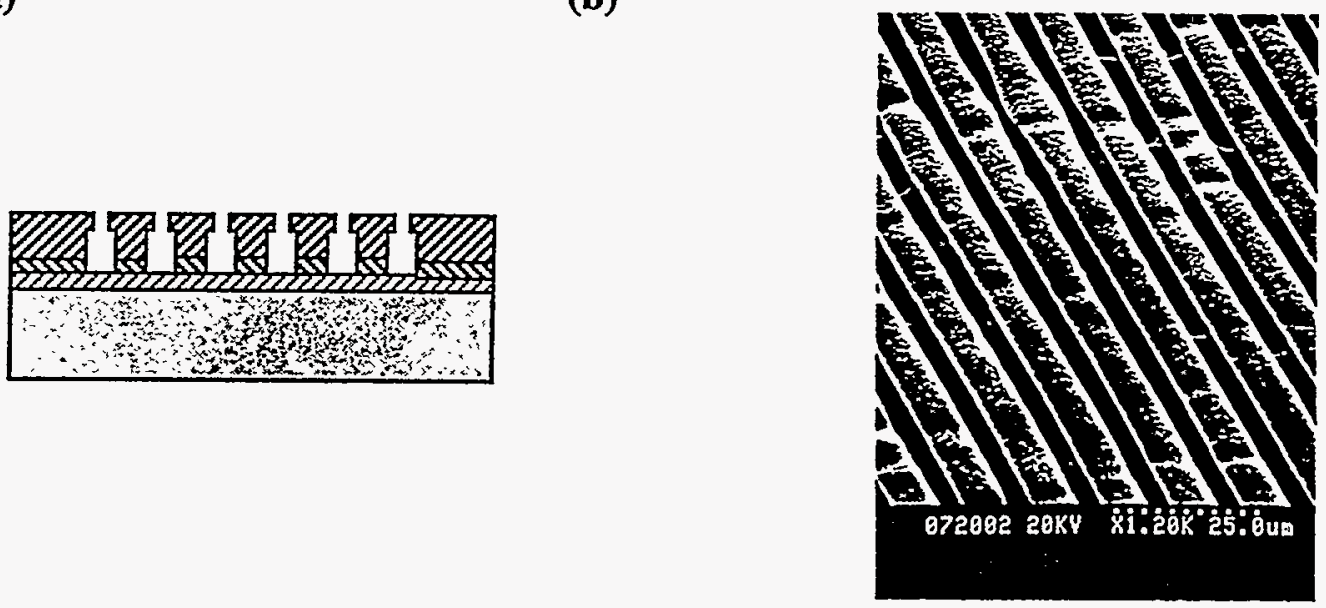

Figure 3: (a) Mushroom effect: electroplated thickness is greater than resist thickness. Resulting space between lines is less than their width. $100 \AA \mathrm{Cr}$ adhesion layer is present. (b) SEM image at $20 \mathrm{kV}$ and magnification indicated.

As a number of difficulties was experienced with materials like nickel, iron and chromium during the manufacturing process we decided on a binary system of copper and carbon. Also, since a greater variety of line widths and duty cycles (other than $5 \mu \mathrm{m}$ width and $5 \mu \mathrm{m}$ gap) was produced with copper and carbon, it was a more desirable test specimen.

Usually, pyrolytic carbon has a polished and almost glassy surface finish which was not the case with the current samples as they exhibited rough and porous spots. Several polishing procedures yielded a surface which did not have optical quality but instead had a number of defects which appeared to be pits. The required thickness for the gridlines was too large to be chemically etched and therefore, an electroplating approach was used as illustrated in figure 2.

(a)

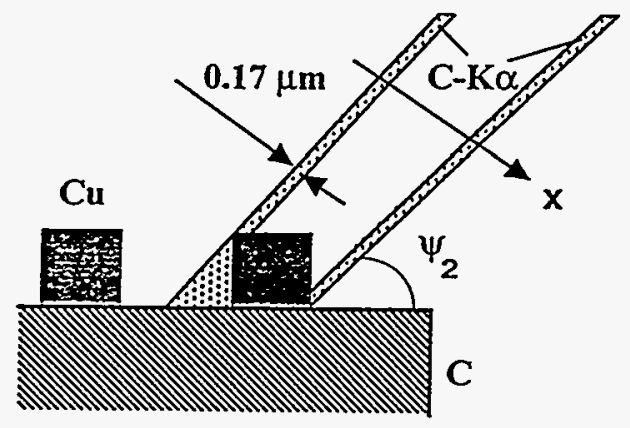

(b)

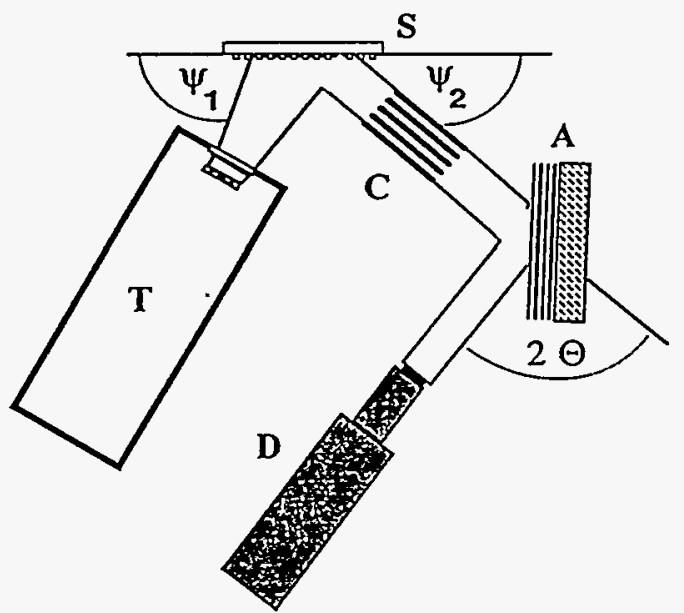

Figure 4: (a) $\mathrm{Cu}$ absorbs $\mathrm{C}-\mathrm{K} \alpha$ almost entirely beyond $0.17 \mu \mathrm{m}$ in $\mathrm{x}$-direction. $\psi_{2}$ is the takeoff angle. (b) Experimental arrangement. $\psi_{1}=63^{\circ}, \Psi_{2}=45^{\circ}, 2 \Theta=43.8^{\circ} @ \mathrm{~d}=60 \AA$. T ... end-window X-ray tube, S ... sample position, $C$... secondary collimator, A ... multilayer analyzer, D ... flow-counter. 
The initial deposition of $100 \AA$ chromium in step (a) acts as an adhesive substrate for subsequent layers. Ideally, the copper in step (c) is electroplated up to a thickness equal to the photoresist thickness. Problems that could possibly be experienced during the manufacturing process include printing of the photoresist pattern having large line width and height. Overplating causes a mushroom effect which eventually results in reduced space between the individual copper lines. Finally, dissolving or etching the $100 \AA$ chromium layer will be difficult if a passivating oxide layer is formed. Figure 3 depicts the cross-section of a problematic sample (a), and (b) a SEM image of a specimen that could be used in the experiments.

As illustrated in figure $4 \mathrm{a}$, there is almost no semi-shading effect observed using the above described sample geometry. Published absorption data for carbon ${ }^{10}$ indicate that no transmission of C-K $\alpha$ fluorescence photons can be detected beyond approximately $0.17 \mu \mathrm{m}$ in the x-direction which corresponds to $0.25 \mu \mathrm{m}$ in the horizontal dimension.

\section{EXPERIMENTAL SETUP AND ANALYZER CHARACTERIZATION}

For the actual measurements a specially equipped Siemens sequential X-Ray spectrometer SRS 303 AS was used. Therein the specimens are irradiated by an end-window X-ray tube with a rhodium target which is separated from the spectrometer chamber by a thin beryllium window with a thickness of 76 micrometers. The selection of the target material provided the continuous Bremsstrahlungsspectrum as well as the characteristic $R h$ lines and in particular at the low energy side of the spectrum the L-lines at $2.69 \mathrm{keV}$. The geometrical arrangement is depicted in figure $4 \mathrm{~b}$. No primary beam filter was used and the secondary collimator ensured a beam divergence of $3^{\circ}$. As a detection system we employed a gas filled (10\% Argon, $90 \%$ Methane) proportional counter with a $1 \mu \mathrm{m}$ polypropylene foil as an entrance window.

Figure 5 shows measurement results for two significant samples of characterized analyzer crystals which exhibit distinctly different performance in this long wavelength region. The specimens were cut from the center of a coated 4 inch silicon wafer (cut $\sim 2^{\circ}$ off $<111>$ ) with a thickness of $500 \mu \mathrm{m}$ and have lateral dimensions of 2.75 inches in length by 1 in width. They were fixed to a $\mathrm{SiO}_{2}$ glass substrate which in turn was mounted onto a support frame. The compact assembly was then inserted into the analyzer position in the spectrometer (A in figure $4 \mathrm{~b}$ ) with a pure element carbon sample (graphite) in place at $S$. The spectra were recorded at tube parameters of $30 \mathrm{kV}$ and $80 \mathrm{~mA}$ (total power of $2.4 \mathrm{~kW}$ ) with $0.05^{\circ}$ per step on the goniometer and a counting time of 1 second per step.
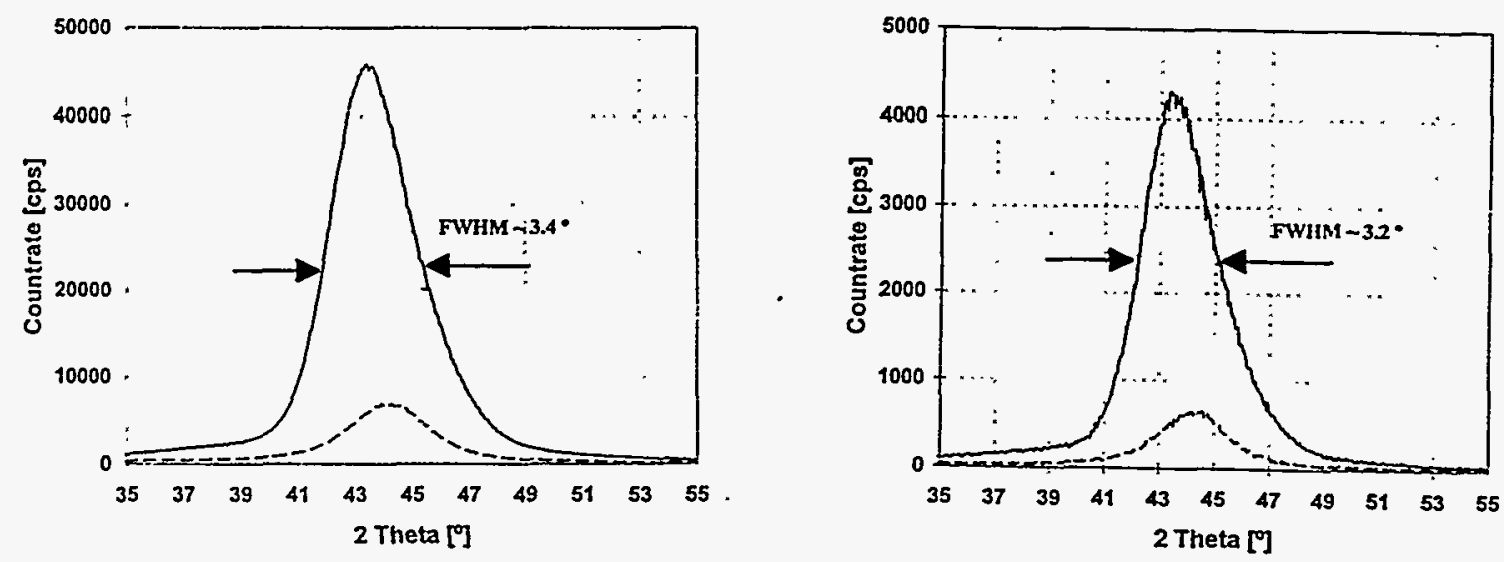

Figure 5: left: Graphite with various analyzers and coarse collimator (divergence $\sim 3.0^{\circ}$ ). Dashed line: WC/C, solid line: V/C. right: Graphite with various analyzers and fine collimator (divergence $\sim 0.7^{\circ}$ ). 
Table II gives the summarized coating variables for the layered structures. They both have the same number of layer pairs but different layer thicknesses and $\Gamma$-values and different $2 \Theta$ peak positions at given carbon $\mathrm{K} \alpha$ wavelength ${ }^{11}$. The FWHM values of the peaks vary between $3.4^{\circ}$ with the coarse collimator and $3.2^{\circ}$ with the fine collimator for the V/C sample, and between $3.2^{\circ}$ with the coarse collimator and $2.8^{\circ}$ with the fine collimator for the WC/C sample (not shown in figure 5). Moreover, there is a remarkable difference in peak reflectivity, which amounts to a factor of 6.64 with coarse collimation and a factor of 6.54 with fine collimation.

\begin{tabular}{ccccc}
\hline & $2 \mathrm{~d}[\AA]$ & $2 \Theta\left[^{\circ}\right]$ & $\mathrm{N}$ & $\Gamma$ \\
\hline WC/C & 118.5 & 44.3 & 60 & 0.25 \\
V/C & 120 & 43.8 & 60 & 0.45 \\
\hline
\end{tabular}

Table II: Coating parameters for multilayer component materials as stated. $\mathrm{N}$... number of layer pairs. $\Gamma$... ratio of high density layer thickness and multilayer period (d spacing).

\section{MEASUREMENT RESULTS AND DISCUSSION}

Countrates from various copper grids on top of pyrolytic carbon substrates as well as from the pure elements carbon and copper have been measured. The orientation of the gridlines was chosen parallel to the primary beam in order to achieve maximum $\mathrm{C}-\mathrm{K} \alpha$ countrate. Appropriate background subtraction was applied and the results for a $6 \mu \mathrm{m}$ linewidth grid with reference to the pure element countrate are shown in figure 6a. The top two curves represent the measured and fitted data for carbon $\mathrm{K} \alpha$, the center curves represent measured and fitted $\mathrm{Cu}-\mathrm{K} \alpha$ data, and the bottom curves relate to trend and measured values for $\mathrm{Cu}-\mathrm{L} \alpha$. The absolute increase in countrate ratio with increasing energy (acceleration voltage) by considering statistical variation is on the order of $4.7 \%$ which cannot be explained by contributions from conventional secondary excitation effects taken into account in the standard FP model. The copper countrate ratios, however, decrease with increasing acceleration voltages which can be attributed to significantly higher self absorption in the sample as the primary radiation is penetrating much further at higher energies.

(a)

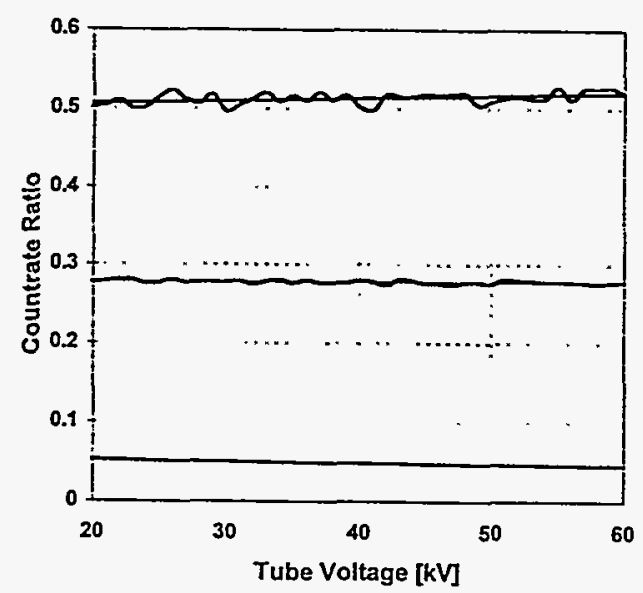

(b)

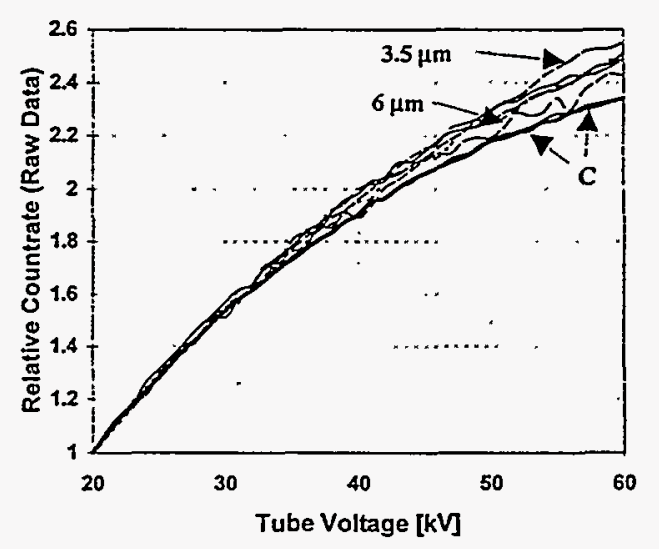

Figure 6: (a) Relative counts from $6 \mu \mathrm{m}$ Cu grid. Top: C-K $\alpha$ measured and fit. Center: $\mathrm{Cu}-\mathrm{K} \alpha$ measured and fit. Bottom: $\mathrm{Cu}-\mathrm{L} \alpha$ measured and fit. (b) $\mathrm{C}-\mathrm{K} \alpha$ counts from various grids. 

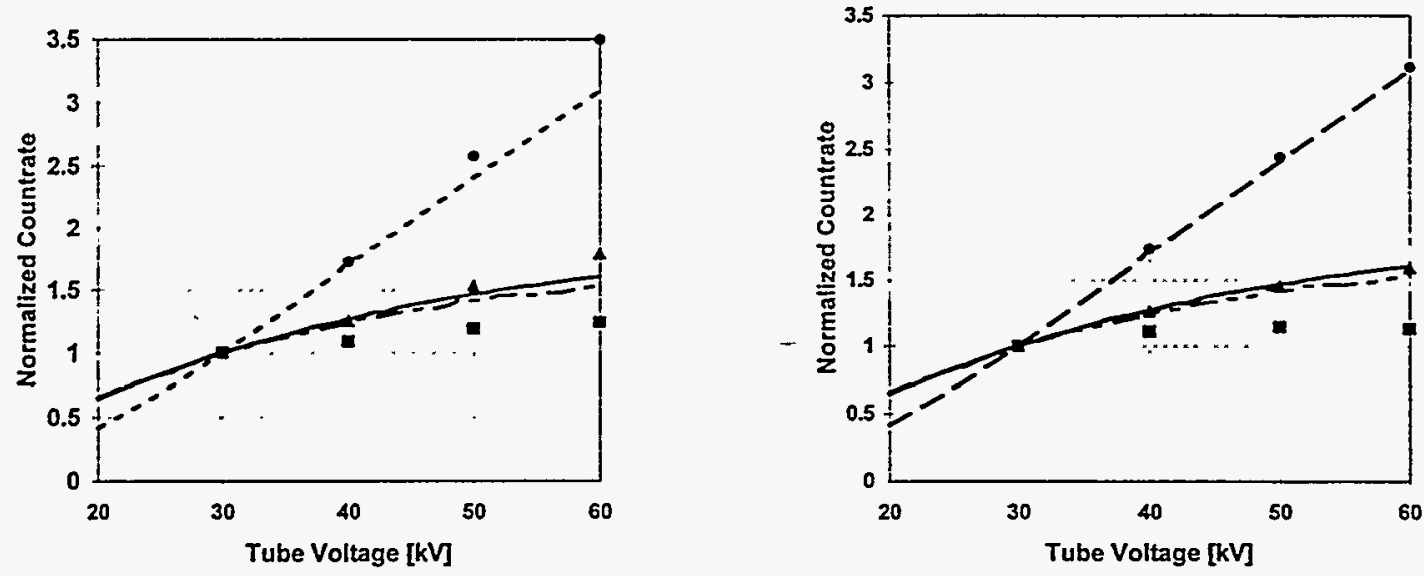

Figure 7: (a) Experiment vs. theory: normalized countrates for $\mathbf{C}$ (measured: squares, calculated: dasheddotted line), $\mathrm{Cu}-\mathrm{K} \alpha$ (measured: circles, calculated: dashed line) and $\mathrm{Cu}-\mathrm{L} \alpha$ (measured: triangles, calculated: solid line). (b) Correction factor obtained from $\mathrm{Cu}-\mathrm{K} \alpha$ and $\mathrm{Cu}-\mathrm{L} \alpha$, respectively, is applied.

Figure $6 \mathrm{~b}$ exhibits the raw data for various grids with linewidths of $3.5 \mu \mathrm{m}$ and $6 \mu \mathrm{m}$ as well as two different graphite specimens. The countrate reference was chosen at a tube voltage of $20 \mathrm{kV}$. Thinner grid linewidths give higher normalized countrates.

A comparison between theoretically obtained results which include photoelectron contribution to secondary exciation of $\mathrm{C}-\mathrm{K} \alpha$ fluorescence radiation and experimental data is presented in figure 7 . Data is normalized to 1 at $30 \mathrm{kV}$ tube voltage.

\section{CONCLUSIONS AND FUTURE WORK}

Binary systems consisting of carbon and copper with very well known absorption geometries have been carefully prepared and measured by means of XRFA. Measurement results show that the C$\mathrm{K} \alpha$ countrates emitted from $\mathrm{Cu}$ grids increase more rapidly with increasing energy than the $\mathrm{C}$ pure element countrate does. A similar effect can be obtained from calculations described in equations 1 through 7 which include secondary excitation effects due to photoelectrons. However, a discrepancy on the order of at least $30 \%$ between theory and experiment was found if the influence of photoelectrons is not properly taken into account. Predictions based on the standard FP model reveal that conventional secondary excitation due to $\mathrm{Cu}-\mathrm{K} \alpha$ and $\mathrm{Cu}-\mathrm{L} \alpha$ fluorescence radiation is negligible. Therefore, we propose that the (energy dependent) photoelectron contribution to secondary excitation of $\mathrm{C}-\mathrm{K} \alpha$ is responsible for the observed increase in $\mathrm{C}-\mathrm{K} \alpha$ countrate compared to the pure element. Also, the computed photoelectron contribution for the presented specimens amounts to a total of about $30 \%$ but the change with energy is rather small.

As additional experiments in that series we plan to manufacture and study grids with appropriate geometries employing different materials such as iron, nickel and chromium. Experiments with grids exhibiting the reversed structure ( $C$ arrays on top of medium $\mathrm{Z}$ substrates) should provide comprehensive information on penetration and interaction of photoelectrons in the low $\mathrm{Z}$ material on top of the substrate. Work has already been done to apply the modified mathematical model to 
multiple thin film layer analysis with results being published elsewhere ${ }^{12}$. Monte Carlo calculations to examine photoelectron behaviour in interface layers containing ultralight elements are currently under way.

\section{ACKNOWLEDGEMENTS}

The authors gratefully acknowledge the technical support from Jim Cox and Ken Haney of the NOVA Operations Group. One of us (F. W.) wants to thank the Austrian "Fonds zur Förderung der wissenschaftlichen Forschung" for financial support. Part of this work has been performed under the auspicies of the United States Department of Energy by the Lawrence Livermore National Laboratory, contract W-7405-ENG-48.

\section{REFERENCES}

1. M.Mantler: Advances in X-ray Analysis 36, 27 (1993).

2. J.Sherman: Spectrochim.Acta 7, 283 (1955).

3. N.Broll, P.Caussin, and M.Peter: X-ray Spectrom. 21, 43 (1992).

4. T.Shiraiwa, and N.Fujino: Jpn J.Appl.Phys. 5, 886 (1966).

5. T.Arai, T.Shoji, and K.Omote: Advances in X-ray Analysis 29, 413 (1986).

6. M. Green and V. E. Cosslett: Proc. Phys. Soc. 78, 1206 (1961).

7. F. Weber: Dissertation and citations therein, Technische Universität Wien (1993).

8. K. N. Stoev: J. Phys. D: Appl. Phys. 25, 131 (1992).

9. F. Weber, M. Mantler and L. Wariwoda: Advances in X-ray Analysis 36, 41 (1993).

10. B. L. Henke, P. Lee, T. J. Tanaka, R. L. Shimbabukuro, and B. K. Fujikawa: Atomic Data and Nucl. Data Tables 27 (1), 27 (1982).

11. J. A. Bearden, and J. S. Thomsen: AIP Handbook (D. E. Gray, ed.), 7-96, Mc Graw Hill (1972).

12. M.Kaufmann, M.Mantler, F.Weber: Advances in X-ray Analysis 37, 205-212 (1994). 
Technical Information Department - Lawrence Livermore National Laboratory University of California - Livermore, California 94551

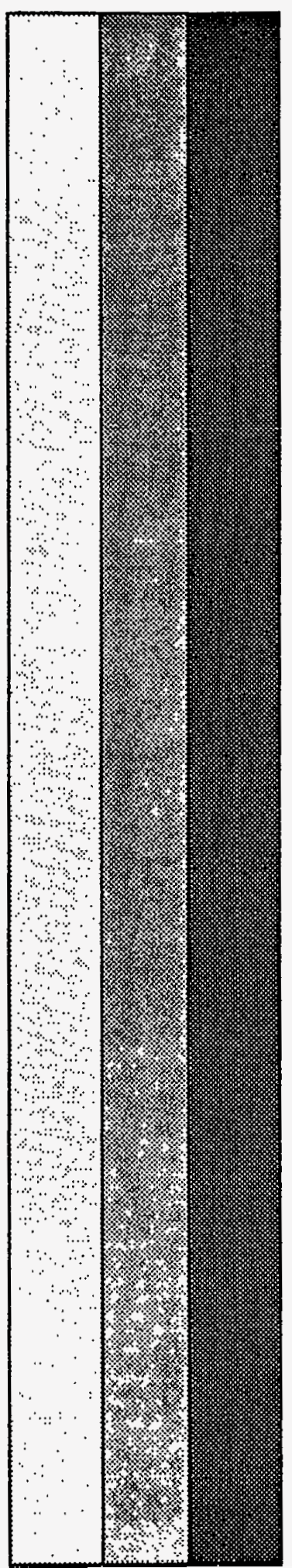

